\title{
A New Fast Sub-Optimal Search Method for Closed-Loop Transmit Diversity System with Limited Number of Feedback bits
}

\author{
S. C. Ip, Z. Zhang, S. W. Cheung and T. I. Yuk \\ Department of Electrical and Electronic Engineering, The University of Hong Kong, Pokfulam, H.K. \\ scip@eee.hku.hk, zhizhang@eee.hku.hk, swcheung@eee.hku.hk, tiyuk@eee.hku.hk
}

\begin{abstract}
In this paper, we present a fast sub-optimal searching algorithm for the closed-loop transmit diversity scheme used in wireless system. Under the low mobility condition and with perfect channel knowledge at the receiving end, our proposed searching algorithm can use a limited number of feedback bits to provide a sub-optimal solution that can achieve the SNR values close to the optimal solution with a greatly reduced complexity and outperform other sub-optimal schemes. The scheme also works well under the presence of feedback errors.
\end{abstract}

\section{INTRODUCTION}

The increasing demand for wireless services makes the capacity and service quality the main concerns of the wireless communication systems. Antenna diversity can play an important role in these and can dramatically enhance the performance of the wireless networks in a fading environment. This technology can be applied to both uplink (Receive Diversity) and downlink (Transmit Diversity) transmissions. However, due to the cost and size limitations, the application of receive diversity to remote units is recently not feasible. While the base station end can afford to have the high complexity and thus, transmit diversity can be applied. As a result, a substantial improvement in the reception quality can be achieved by using more antennas at the transmitting end.

Open-loop and closed-loop are two classifications of transmit diversity in $W$-CDMA [1], which has already been included in the $3 \mathrm{G}$ wireless standard. The closed-loop scheme provides feedback information for the transmit antennas while the open-loop does not. In the earliest stage, the open loop diversity schemes were all simple in configuration by transmitting the same symbol at reduced power simultaneously through different antennas and keep the total power unchanged. Space-Time Transmit Diversity (STTD) using the technique described in [2] is an open-loop transmit diversity scheme adopted by the Third Generation Partnership Project (3GPP) to maximize the diversity gain. The open-loop scheme ensures that the complexity of the mobile station receiver is always kept low while no signaling overhead is required. Since the scheme does not take into account the fact that the channel is time-varying, this may cause substantial degradation to the $S N R$ at the receiving end since no adaptation is made at the transmitting end. However, this could be overcome by using the closed-loop diversity scheme which takes into account the time-varying properties of a real practical channel.

Feedback has been proposed many years ago and now it is a mean to obtain advantage in diversity. Selection diversity in [3] is a common example of feedback in transmit diversity system. The closed-loop transmit diversity scheme with the feedback of the weight vector was first proposed by Gerlach and Paulraj in 1994 [4], in which the receiving end calculates the optimal weight vector which contains the weight of each transmit antenna based on the channel gain information from the receiving end and the calculated value will then be fed back to the transmitting side. With the help of this channel side information, the closed-loop diversity scheme should typically out-perform the open-loop one under a lowmobility environment in which the delay of feedback signaling does not exceed the coherence time of the channel.

Under a real practical environment, bandwidth is always limited, thus, the partial channel feedback has been investigated [5]. Though the number of feedback bits for each antenna is always limited, there should still exist an optimal quantized transmit weight vector which could achieve the maximum $S N R$ value under different varying fading channel environments. However, complexity could be problem because it increases exponentially with the number of transmit antennas. Here we propose a fast searching algorithm in which the complexity, the average distance and the differences between the SNRs of the optimal scheme and those of the proposed scheme under the limited quantized feedback bit condition are studied. Simulations results show that our proposed method can yield a $S N R$ value closed to that of the optimal scheme, but a lower complexity under both error free and feedback error conditions. The results also show that, with different numbers of transit transmit antennas, the deviation of the $S N R$ from that of the optimal scheme is still small. Thus, our proposed scheme can enjoy an excellent $S N R$ performance while at the same time the work load of the receiving end in transmit weight vector calculation is greatly reduced. 
This paper is organized as follows: Section II presents the transmit diversity system model. Our proposed transmit diversity scheme and its complexity are described in Section III. The simulation results in different environment conditions and the comparison with other sub-optimal searching scheme are given in Section IV. Section V concludes the results.

\section{SYSTEM MODEL}

Assume the system operates in Rayleigh Fading Channel throughout the paper. The channel coefficients are accurately estimated at the receiving end and there is no feedback delay between the transmitting and the receiving ends. The antennas are spaced far enough apart so that the signals can be assumed to undergo independent fading and we also assume that the frame duration is much less than the channel coherence bandwidth. Consider a system with $M$ transmit antennas and one receive antenna, the transmitted signal can be expressed as:

$$
X=\sqrt{P} d W
$$

where $P$ is the total transmit power, $d$ is the data symbol, $X=\left[x_{1}, x_{2}, \ldots \ldots x_{M}\right]^{T}$ is the transmit signal vector and $W=\left[w_{1}, w_{2}, \ldots \ldots w_{M}\right]^{T}$ is the transmit weight vector which is of unit norm. The channel gain is modeled by a $1 x M$ matrix $G$ which is complex and contains $g_{i}$ which represents the independent complex gain of the signal from transmit antenna $i$ to the receiver with zero mean and unity standard deviation. The received signal could be expressed as follows:

$$
Y=G X+N
$$

where $n$ is a white Gaussian noise vector with independent complex Gaussian random variable elements having zero mean and standard deviation $\sigma_{n}$. If maximal ratio combination is used at receiver, the signal-to-noise ratio is:

$$
S N R=\frac{P}{\sigma_{n}^{2}} W^{H} G^{H} G W
$$

With an infinite number of feedback bits, the maximum SNR can be obtained by choosing a suitable $W$ which is the eigenvector associated with the maximum eigenvalue of the Hermitian matrix $G^{H} G$. However, under the limited bandwidth condition, the number of feedback bits is finite and the transmit weight $w$ has to be quantized before they are being fed back to the transmitting end. Assuming that two feedback bits are used for each transmit antenna, the weight $w$ is one of the elements of the quantization set specified in product form below:

$$
w=\prod_{m=1}^{M}\left\{e^{j 2 \pi(k-1) / 4} / \sqrt{M}, k=1,2,3,4\right\}
$$

To obtain an optimal quantized weight vector for maximizing the $S N R$, a complete search through the above set is required by substituting each of the value to equation (3). This could yields a complexity of the order $4^{M}$ and the value will get higher with increasing number of transmit antennas.

\section{PROPOSED SCHEME}

The proposed algorithm can be used with any number of antennas. However, for simplicity in explanation of the algorithm, we first start with the system which has 2 transmit antennas. The channel gain of each transmit antenna is being modeled as a vector in a complex plane. A weight vector is produced using the designed searching algorithm and the complex vector is rotated according to the corresponding elements in the weight vector so that the final combined vector has the maximum magnitude and thus the maximum $S N R$. Under two feedback bits condition, each vector is allowed to rotated by $z * \pi / 2$ where $z=0,1,2,3$, before it is combined with other vectors. Figure 1 illustrate the case when $M=2$,

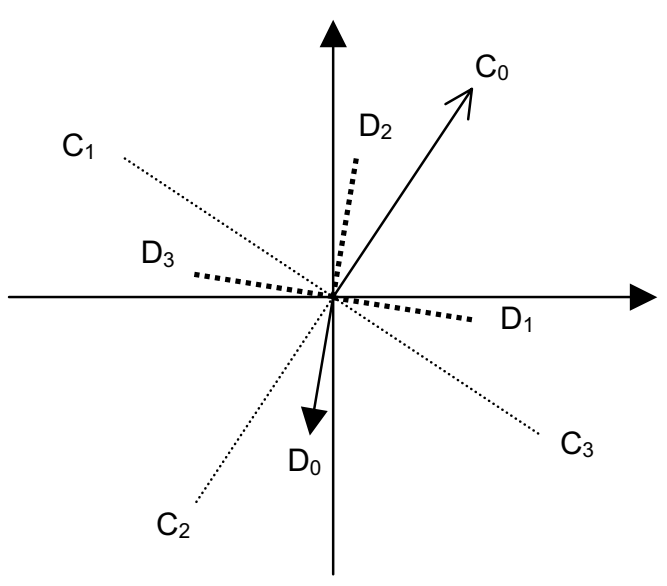

Fig. 1. $\quad M=2$ case

$C_{0}$ and $D_{0}$ are the original complex channel gain elements. The vector should be rotated so as to obtain the smallest angle difference. With 2 feedback bits, there should exist four groups of optimal vector combination such that the angle difference between the vectors is less than or equal to $\pi / 4$. Thus, there exist four groups of optimum vector combination: $C_{0}$ and $D_{2} ; C_{3}$ and $D_{1} ; C_{2}$ and $D_{0} ; C_{1}$ and $D_{3}$. Each of the above combinations will form the final vector with maximum magnitude and hence the maximum SNR. This approach can be extended to be used the in multiantenna case as described below. 


\section{A. Multi-antenna case}

The complex channel vector with the largest magnitude among the $M$ vectors is first selected among all the vectors. Its neighboring vectors are then rotated so that they are within $\pi / 4$ of the selected vector. The vector with the smallest angle difference with the selected vector is combined with the selected vector. These two vectors are then discarded from the complex plane. Next, the remaining vectors are rotated to within $\pi / 4$ of the newly combined vector which is again combined with the closest neighboring vector to form a new vector. The process continues until the final single vector is obtained. Figure 2 illustrates an example with $M=4$ :

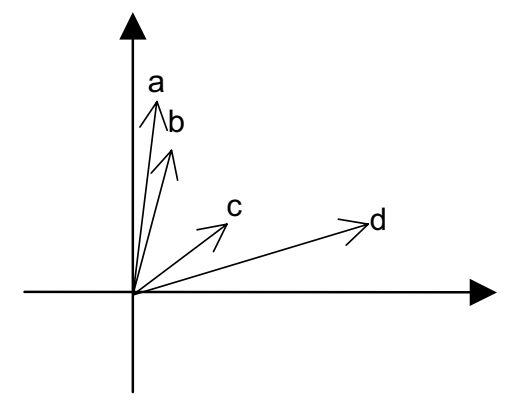

Fig 2(a) Step 1. Choose the vector with the largest magnitude

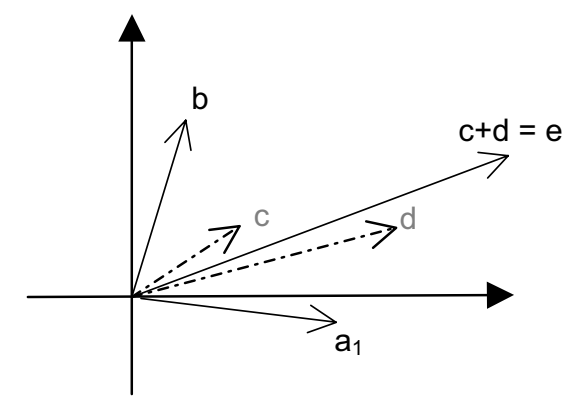

Fig. 2(b) Step 2. Rotate the neighbor vectors and combine with the first chosen vector

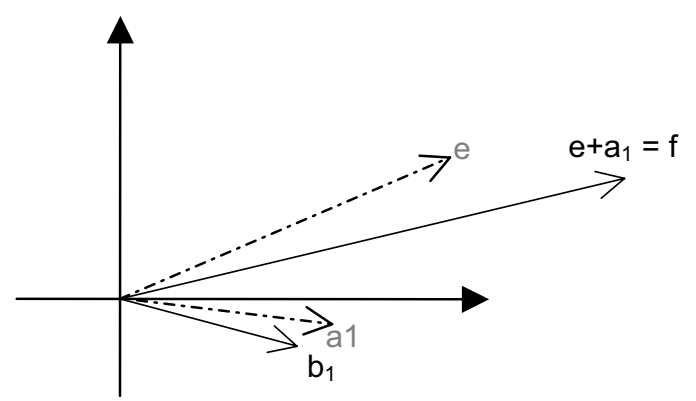

Fig. 2(c) Step 3. Rotate the remaining vectors and combine with the newly formed vector
Fig. 2a-2c shows the steps in combining the complex Gaussian channel vectors. In Fig. 2a, vector $d$ has the largest magnitude and so is first selected. The remaining vectors are then allowed to rotate until a vector which has the smallest angle difference and within $\pi / 4$ of vector $d$. Since vector $c$ is the closest vector to $d$, it is combined with vector $d$ to form a new vector $e$ as shown in Fig. 2b. The comparison procedure continues. Vector $a$ rotates $\pi / 2$ clockwise to give vector $a_{l}$ so that it is within $\pi / 4$ of vector $e$ and becomes the closest neighboring vector to $e$. The two vectors $e$ and $a_{l}$ in Fig. 2b are then combined to form a new vector $f$. Finally, vector $b$ rotates $\pi / 2$ clockwise in Fig. $2 \mathrm{c}$ to give $b_{1}$ which is within $\pi / 4$ of vector $f$. The two vectors then combine to form the final vector.

\section{B. Complexity of the proposed scheme}

Assume there are $M$ complex channel vectors. Since the first step is to find the vector with the maximum magnitude, there are $(M-1)$ comparisons. After the first new vector is formed, the remaining (M-2) vectors are comparing with the newly combined vector in order to select the one with the smaller angle difference. Thus, this obtains a number of $(M-$ 2) comparisons. The procedure is iterated until the final vector is formed. Since the vectors are rotated to find the angle difference, the number of comparisons equals to $(M-1)$ $+2(M-2)(M-1)=(M-1)(2 M-3)$, for $M \geq 3$. So the complexity is much lower than that of the optimal scheme.

\section{SIMULATION RESULTS}

Simulation tests on the $S N R$ performances of our proposed searching algorithm have been performed with different numbers of transmit antenna cases under both error free and erroneous conditions. The number of feedback bit for each transmit antenna is restricted to two in all cases. Difference in $\operatorname{SNR}\left(S_{i}\right)$ and the average distance $\left(D_{i}\right)$ between the proposed algorithm and the optimal solution are studied and we also compare the result with other sub-optimal algorithm i.e. the co-phasing algorithm [6]. The results are shown in Tables 1-4.

\begin{tabular}{|l|l|l|l|l|}
\hline & $M=3$ & $M=4$ & $M=5$ & $M=6$ \\
\hline$S_{i}<0.01$ & $99.47 \%$ & $98.21 \%$ & $97.15 \%$ & $95.72 \%$ \\
\hline$D_{i}$ & $\begin{array}{l}2.0212 \mathrm{e}- \\
004\end{array}$ & $\begin{array}{l}8.7495 \mathrm{e}- \\
004\end{array}$ & 0.0017 & 0.0028 \\
& 004 & \\
\hline
\end{tabular}

Table 1. Performance of proposed method with no feedback error

\begin{tabular}{|l|l|l|l|l|}
\hline & $M=3$ & $M=4$ & $M=5$ & $M=6$ \\
\hline$S_{i}<0.01$ & $99.15 \%$ & $98.14 \%$ & $96.56 \%$ & $95.23 \%$ \\
\hline$D_{i}$ & 0.0035 & 0.0053 & 0.0087 & 0.0101 \\
\hline
\end{tabular}

Table 2. Performance of proposed method with $B E R=10^{-3}$ for each antenna 


\begin{tabular}{|l|l|l|l|l|}
\hline & $M=3$ & $M=4$ & $M=5$ & $M=6$ \\
\hline$S_{i}<0.01$ & $84.58 \%$ & $72.35 \%$ & $63.388 \%$ & $56.12 \%$ \\
\hline$D_{i}$ & 0.0331 & 0.0737 & 0.1067 & 0.1410 \\
\hline
\end{tabular}

Table 3. Performance of Co-Phasing Algorithm with no feedback error

\begin{tabular}{|l|l|l|l|l|}
\hline & $M=3$ & $M=4$ & $M=5$ & $M=6$ \\
\hline$S_{i}<0.01$ & $84.08 \%$ & $71.78 \%$ & $62.86 \%$ & $55.54 \%$ \\
\hline$D_{i}$ & 0.0361 & 0.0776 & 0.1103 & 0.1516 \\
\hline
\end{tabular}

Table 4. Performance of Co-Phasing Algorithm with $B E R=10^{-3}$ for each antenna

Table 1 shows that the proposed method gives the $S N R$ values close to the optimal $S N R$ as indicated by the small percentage difference in $S$, while most of the $S N R$ differences fall within $S_{i}<0.01$. This is the case with no feedback error.

In fact, the proposed method also works well in error condition. Table 2 shows the performance of the scheme with feedback error, where the $B E R$ equals to $10^{-3}$ for each antenna and Gray coding is used for the feedback bits. The $S N R$ performance is nearly the same as that with no feedback error case. Although the performance on average distance is a slightly higher, it still keeps relatively low.

Tables 3 and 4 show the performances of the co-phasing algorithm [6]. The $S N R$ difference of the scheme with $S_{i}<0.01$ is lower than that of our proposed method and it becomes obvious in the cases with more antennas. The above results show that our proposed method outperforms the cophasing sub-optimal method by more than $30 \%$ in $S N R$ difference when $M \geq 5$ under both error free and erroneous conditions. The average distance between the optimal solution and the co-phasing algorithm is at least ten times higher in both cases.

\section{CONCLUSION}

Optimal searching algorithm is seldom used in real case due to the high complexity, thus sub-optimal solution with less complexity is needed. This paper has introduced a fast sub-optimal searching algorithm for obtaining the weight vectors of the closed-loop transmit diversity scheme under a limited feedback bits condition.

It has been shown that the proposed methods could achieve a $S N R$ performance close to that of the optimal solution with limited number of feedback bit and it works well in both error free and erroneous conditions. Both of its $S N R$ and average distance performance are superior to the other sub-optimal method such as co-phasing especially in the cases with more antennas. This proposed method also has an improvement over our previous proposed sub-optimal searching algorithm in [7].

\section{REFERENCES}

[1] 3GPP, TS 25.211 v3.10.0(R99), Physical channels and mapping of transport channels onto physical channels (FDD)

[2] S.M.Alamouti, "A Simple Transmit Diversity Technique for Wireless Communications", IEEE JSAC, vol. 16, pp.1451-58, Oct. 1998,

[3] W. C. Jakes, Microwave Mobile Communications. John Wiley an dSons, New York, 1974

[4] Derek Gerlach and Arogyaswami Paulraj, "Adaptive Transmitting Antennas with Feedback", IEEE Signal Processing Letters, Oct, 1994

[5] Robert W. Heathand Arogyaswami Paulraj, "A Simple Scheme for Transmit Diversity Using Partial Channel Feedback", Signals, Systems \& Computers, 1998. Conference Record of the Thirty-Second Asilomar Conference on, Volume 2, 1-4 Nov, 1998

[6] J.Hämäläinen, R. Wichman, "Performance Analysis of Closed-Loop Transmit Diversity in the Presence of Feedback Errors", IEEE Proc. PIMRC, 2002.

[7] S.C. Ip, Z. Zhang, S.W. Cheung, T.I. Yuk, "Novel Search Algorithms for Closed-loop Transmit Diversity System with Limited Number of Feedback Bits", IEEE VTC fall, 2004 